\section{Diagnostic values of microRNA $27 a$ in breast cancer patients}

\author{
Mohamed I Seddik ${ }^{1}$, Osama Osman ${ }^{1}$, Murad A \\ Jabir $^{2}$, Eman M Abdelrahman ${ }^{1}$, Dalia A Nigm ${ }^{1}$
}

${ }^{1}$ Department of clinical pathology, Faculty of medicine, Assiut University, Assiut, Egypt.

${ }^{2}$ Surgical oncology department, South Egypt cancer institute, Assiut University, Assiut, Egypt.
The Egyptian Journal of Immunology Volume 28 (3), 2021: 127-137. www.Ejimmunology.org
Corresponding author: Eman $M$ Abdelrahman, Clinical Pathology Department, Faculty of Medicine, Assiut University, Assiut, Egypt.

Email: dr.emanmohammed@yahoo.com.

\begin{abstract}
Breast cancer is one of the most malignant tumors in women across the globe. Diagnosis of breast cancer at early stages is essential to improve treatment outcomes and decrease mortality rates. There is a pressing need for new non-invasive biomarkers to improve early diagnosis of breast cancer. This study aims to assess plasma miR-27a for the early diagnosis of breast cancer. miR-27a was evaluated in a total of 95 blood samples, 40 newly diagnosed cancer patients, 20 patients with benign breast lesions, 20 females with positive family history for breast cancer and 15 apparently healthy controls, using quantitative real time polymerase chain reaction. Our results exhibited significantly higher expression level of plasma miR-27a in breast cancer patients (median= 8.3 and 19 fold change for early and late stages respectively) compared to controls, high risk group and benign group with $(P<0.001)$ for each. Plasma miR-27a was significantly higher in late breast cancer (median=19 fold change) compared to early breast cancer (median= 8.3) with $(P<0.001)$. There was no statistically significant difference of plasmamiR-27a levels in benign group (median=1.8 fold change) compared to both control group and high risk group. There was no statistically significant difference of plasma miR-27a levels in high risk group (median= 1.2 fold change) compared to control group (median= 1 fold change). We performed Receiver Operating Characteristic (ROC) analysis for discriminating malignant from non-malignant cases. Plasma miR-27a yielded an area under the curve (AUC) of 0.983 with sensitivity $97.5 \%$, specificity $91 \%$ and accuracy $94 \%$. We concluded that miR-27a expression level represents sensitive and specific non-invasive molecular biomarkers for diagnosis of breast cancer.
\end{abstract}

Keywords: Breast cancer, Molecular biomarkers, Circulating MiRNAs.

Date received: 15 January 2021; accepted: 22 May 2021

\section{Introduction}

Breast cancer (BC) is the most common cancer in women and the second most frequent cancer worldwide. ${ }^{1}$ In 2018, breast cancer accounted for about 2.1 million cancer cases worldwide, and is considered the fifth leading cause of cancer related deaths. ${ }^{2}$

In Egypt, BC represents $18.9 \%$ of total cancer cases (32.04\% in women and $2.2 \%$ in men). The earlier the condition is found, the better the chances of surviving it. In early stage, breast 
cancer has a $97 \%$ chance of surviving 5 years. However, the likelihood of a woman surviving 5 years drops to $20 \%$ once it spreads to other parts of the body. ${ }^{3}$ The disease is usually diagnosed at an advanced stage among the Egyptian women. The majority of cases are diagnosed between the ages of 30-60 years. ${ }^{4}$

Previous studies have suggested that early $\mathrm{BC}$ detection with suitable management could significantly decrease $\mathrm{BC}$ mortality rates in the long-term. ${ }^{5}$ Mammography is the present standard breast screening technique. ${ }^{6}$ In recent years, ultrasound has been used as an additional imaging tool for diagnosis of $B C$ together with mammography. ${ }^{7}$ Magnetic resonance imaging has the ability to detect small lesions that cannot be identified by mammography. ${ }^{8}$ Breast biopsies are performed to distinguish between malignant and benign tissue lesions, but this is an invasive method and requires qualified personnel. ${ }^{9}$ Biomarker-based methods such as radioimmunoassay, immunohistochemistry, immunosorbent assay (ELISA) and fluoroimmunoassay also offer diagnostic methods for $\mathrm{BC}$ diagnosis. ${ }^{10}$

Serum tumor markers are soluble molecules released into the blood stream by malignant cells or other cell types of tumor microenvironment. The measurement of these molecules in blood provides an economical and yet a non-invasive methods for diagnosis of the presence of $B C$ as well as its progression. In case of $B C$, different serum biomarkers were tried for these purposes, the most commonly used are carcinoembryonic antigen (CEA), the cancer antigen 15-3 (CA15-3), circulating cytokeratins such as tissue polypeptide antigen and cytokeratin 19 fragment, and the human epidermal growth factor receptor $2 .^{11}$

MicroRNAs (miRNAs) are a class of endogenous, small, non-coding RNAs which are 21 23 nucleotides in length. They are involved in many human cancers and can either act as oncogenic miRNAs (oncomiRs) or tumor suppressor miRNAs. ${ }^{12}$

Circulating miRNAs have been found in peripheral blood and other body fluids, such as tears and urine. Like tissue miRNAs, altered profiles of circulating miRNAs were found indifferent pathological conditions including cancers. ${ }^{13}$ Because miRNAs are stable in body fluids, and easily detected by noninvasive procedures, they are considered an attractive biomarker candidates. ${ }^{14}$

The miRNAs associated with breast cancers include miR-21, miR-155, miR-27a, miR-205, miR-145 and miR-320a. They have roles in BC as diagnostic, prognostic markers and as markers of metastasis. $^{15}$ They may have role in treatment resistance as anticancerous drugs such as Tamoxifen and Trastuzumab. ${ }^{15}$

miR-27 has two isoforms: miR-27a and miR$27 \mathrm{~b}$, they are transcribed from different chromosomes and differ in only one nucleotide. miR-27a is located on chromosome19. Since miR-27a acts as oncogenic miRNA, many studies have been excited to use it in clinical applications as a biomarker to help in the diagnosis and treatment. miR-27a has been used as a diagnostic and prognostic biomarker in several tumors. ${ }^{16}$ It is up-regulated in many tumors including gastric cancer, ${ }^{17}$ liver cancer, ${ }^{18}$ cervical cancer, ${ }^{19}$ ovarian cancer, ${ }^{20}$ and prostatic cancer. $^{21}$

In BC, miR-27a acts as oncogenic miRNA, It is found to stimulate angiogenesis and to enhance the proliferation, migration and invasion of $B C$ cells, so levels of miR-27a expression may have a diagnostic value in $\mathrm{BC}^{22}$ and is considered as an independent prognostic factor for $B C$ progression. $^{23}$

Few studies were conducted to assess the diagnostic performance of this biomarker in peripheral blood of breast cancer patients. This study aimed to evaluate the clinical utility of plasma miR-27a measurement as a potential biomarker in peripheral blood for diagnosis of breast cancer. We evaluated its level in breast cancer patients and in patients with benign breast lesions in comparison with healthy controls. This study also aimed to study the role of measuring MicroRNA 27a in females at risk of getting $B C$ due to positive family history as a non invasive biomarker for early diagnosis of breast cancer, to study the relation of MicroRNA 27a to breast cancer staging and to compare between $\mathrm{miR}-27 \mathrm{a}$ and the routine markers (CEA, CA15-3) as biomarkers for breast cancer diagnosis. 


\section{Patients and Methods}

This study was approved by the Assiut Faculty of Medicine Ethical Committee and informed consent was obtained from each participant before enrollment. This study was conducted on ninety-five female individuals who were admitted to the General surgery Department and Surgical Oncology Department, Assiut University Hospitals. It was conducted during the period from May 2019 to February 2020.

Our study included 15 healthy volunteers, 20 high risk female individuals, 20 patients with benign breast lesions and $40 \mathrm{BC}$ patients.

According to the study design, inclusion criteria were primary BC patients before receiving any treatment or underwent surgical resection and have not reported other malignancies. Our study also included 20 females with benign breast lesions. Patients were diagnosed on the basis of history, clinical examination, laboratory findings, and imaging studies. The tumors were confirmed histologically and staged according to TNM staging, $7^{\text {th }}$ edition, American Joint Committee of Cancer staging system. ${ }^{24}$ Demographic and clinicopathological data for enrolled individuals were collected from their clinical files.

Our study also included 15 healthy volunteers with no history suggestive of breast problems, free clinical examination and no lesions by imaging and they were selected as the healthy control group. Twenty healthy individuals at risk of getting $B C$ as they had positive family history of $B C$ including mothers, sisters or daughters of $\mathrm{BC}$ cases were also included in our study. They were with no history suggestive of breast problems, free clinical examination and no lesions by imaging studies.

The study population was divided into 5 groups; the first group (control group) consisted of 15 apparently healthy controls. The second group (High risk group) included 20 healthy female individuals with positive family history of BC. The third group (benign group) included 20 patients with benign breast lesions. The fourth and fifth groups included 40 patients with BC [stage I /II (early breast cancer), stage III / IV (late breast cancer) respectively]. They were classified into 3 (stage I), 17 (stage II), 13 (stage III) and 7 (stage IV). ${ }^{25}$
Blood samples were taken from those patients before the initiation of any chemotherapeutic or surgical treatment. The laboratory work and interpretations were carried out at Clinical Pathology Department, Assiut University Hospital.

\section{Exclusion criteria}

1. Patients with breast cancer that received chemotherapy, radiotherapy or surgical treatment.

\section{History of malignant tumors in other organs.}

3. Other conditions or medications that may affect the biomarkers included in our study like: liver disorders as cirrhosis, pancreatitis and inflammatory bowel disorders.

\section{Blood samples}

- Serum sample for determination of CEA and CA 15-3.

- $2 \mathrm{ml}$ blood into EDTA coated tube centrifuged at $1000 \mathrm{rpm}$ for $10 \mathrm{~min}$, and then plasma was carefully transferred into an RNase-free tube for extraction of RNA. Plasma was stored at -80 ${ }^{\circ} \mathrm{C}$ until assay.

Laboratory investigations done for all participants:

- Determination of serum carcinoembryonic antigen (CEA) and cancer antigen 15-3 (CA 15$3)$.

- Measurement of plasma miR-27a by quantitative real-time reverse transcription polymerase chain reaction (qRT-PCR).

\section{Determination of CEA}

The procedure is fully automated using the ADVIA Centaur Auto-Analyzer, Siemens Healthineers, USA, Catalog Number: 09788458.

It is a two-site sandwich chemiluminescence immunoassay, which uses constant amounts of two antibodies. The first antibody, in the Lite Phase Reagent, is a purified polyclonal rabbit anti-CEA antibody labelled with acridinium ester. The second antibody, in the Solid Phase Reagent, is a monoclonal mouse anti-CEA antibody covalently coupled to paramagnetic 
particles. Based on the $95 \%$ confidence interval, the reference value is $<5.093 \mathrm{ng} / \mathrm{ml}$.

\section{Determination of CA 15-3}

The procedure is fully automated on ADVIA Centaur Auto-Analyzer, Siemens Healthineers, USA, and Catalog Number: 00128609.

It is a two-site sandwich chemiluminescence immunoassay, which uses constant amounts of two antibodies. The Lite reagent is composed of the monoclonal mouse antibody, DF3, specific for CA15-3, labelled with acridinium ester. The conjugate reagent is composed of the monoclonal mouse antibody 115D8, specific for CA15-3 second antibody, labeled with fluorescin. The Solid Phase is composed of purified monoclonal mouse capture antibody, which is covalently coupled to paramagnetic particles. The sample is incubated with both conjugate reagent and solid phase simultaneously for 20 minutes. After incubation, the immune-complex is washed and the lite reagent is added, incubated for an additional 20 minutes and then washed again. This two-step protocol eliminates high-dose hook effect in this assay. Based on the $95 \%$ confidence interval, the reference value is $<32.4 \mathrm{U} / \mathrm{ml}$.

\section{Determination of plasma miR-27a by ( $q R T-P C R$ )}

It was done using a 7500 fast real time PCR (Applied Biosystems - USA).

\section{Plasma total RNA extraction}

RNA extraction from stored plasma (after thawing) was carried out using miRNeasy Mini Kit (Cat. No. 217004; Qiagen - Germany), As recommended by manufacturer's instructions: Plasma denaturation was achieved QIAzol lysis reagent was added to serum samples (with volume ratio 5:1), after incubation at room temperature for $5 \mathrm{~min}$; phase separation was carried out using one volume of chloroform and centrifugation for $15 \mathrm{~min}$ at $15000 \mathrm{rpm}$ at $4^{\circ} \mathrm{C}$. Ethanol $100 \%$ was added to the upper aqueous phase then $700 \mu \mathrm{l}$ of the sample was pipetted into RNeasy Mini column (Qiagen, USA) and centrifuged for $15 \mathrm{sec}$ at $1000 \mathrm{rpm}$ at room temperature. Two washing buffer solutions supplied with the kit were then used on two consecutive steps (RWT and RPE respectively) to wash the mini spin columns with centrifugation at $10000 \mathrm{rpm}$ for $15 \mathrm{sec}$. at room temperature for each step. Finally, the total RNA was eluted using $50 \mu \mathrm{l}$ of RNase-free water applied directly into the mini spin column silica membrane and centrifuged for $1 \mathrm{~min}$ at $10000 \mathrm{rpm}$ at room temperature.

miRNAs reverse transcription into complementary DNA (cDNA)

Reverse transcription was performed using miScript $^{\circledR}$ II RT Kit (Cat. No.218160; Qiagen Germany), according to the manufacturer' instructions. The reverse RT mix was prepared using $4 \mu \mathrm{l}$ miScriptHiSpec Buffer $(5 x), 2 \mu l$ miScript Reverse Transcriptase Mix, $2 \mu l$ miScript Nucleic Mix (10x), $7 \mu \mathrm{l}$ RNase-free water, and $5 \mu \mathrm{l}$ of the extracted RNA to yield a $20 \mu \mathrm{l}$ final reaction volume. In order to synthesize the cDNAs, the RT mix was placed in thermal cycler under the following conditions: $60 \mathrm{~min}$ at $37^{\circ} \mathrm{C}$ followed by $5 \mathrm{~min}$ incubation at $95^{\circ} \mathrm{C}$.

\section{Quantification of mature miRNAs using qRT-PCR}

Real time PCR was carried out using miScript Primer Assay for miR-27a [Hs_miR-27a*_1 (Homosapien miR-27a) (Cat. No.MS00009240; Qiagen- Germany)] with primer sequence 5'AGGGCUUAGCUGCUUGUGAGCA. U6 small nuclear RNA [Hs_RNU6-2 (Cat. No.MS00033740; Qiagen- Germany)] was used as an endogenous control for data normalization with primer sequence ACGCAAATTCGTGAAGCGTT. The reaction was carried out using miScript SYBR ${ }^{\circledR}$ Green PCR Kit (Cat. No. 218073; Qiagen - Germany).According to the manufacturer' instructions: RNase-free water, template cDNA and the reaction mix were dispensed in the individual wells of the PCR plate. The qRT -PCR was performed using Rotor-Gene Q72-well rotor (Qiagen - Germany) which was programmed for the following conditions: incubation at $95^{\circ} \mathrm{C}$ for $15 \mathrm{~min}$ as a preliminary activation step for HotStarTaq DNA polymerase, followed by 40 amplification cycles, and each cycle is achieved by three consecutive steps of DNA denaturation at $94^{\circ} \mathrm{C}$ for $15 \mathrm{~s}$, annealing at $55^{\circ} \mathrm{C}$ for $30 \mathrm{~s}$, and extension at 
$70^{\circ} \mathrm{C}$ for $30 \mathrm{sec}$. Fluorescence measurement was made at every cycle. ${ }^{26}$

Specific amplification of miRNA was ensured through analyzing the melting curves. Cycle threshold $(\mathrm{Ct})$ value was defined as the cycle number in which there is the first detectable increase in fluorescence signals above a defined threshold. Ct values were automatically calculated. The expression levels of investigated miRNAs were evaluated using $\Delta \mathrm{Ct}$ method (Livak method for relative gene expression analysis) ${ }^{27}$ where ct values for the RNU6 were subtracted from ct values of the target miRNA. This was done for both patients and the control groups. Then, $\Delta \Delta \mathrm{Ct}$ values were calculated by subtracting the mean $\Delta \mathrm{Ct}$ value of the control samples from $\Delta \mathrm{Ct}$ values of the patient samples. Then, the fold change (FC) of expression or relative quantitation for the targeted miRNA was calculated using $2^{-\Delta \Delta C t}$ method. The results were expressed as FC compared to the control sample which was considered the normal value and assumed to equal 1 .

$\Delta \mathrm{Ct}$ Sample $=\mathrm{Ct}_{\text {miR-27a }}-\mathrm{Ct}_{\mathrm{RNU6}}$

$\Delta \mathrm{Ct}$ Control $=\mathrm{Ct}_{\text {miR-27a }}-\mathrm{Ct}_{\mathrm{RNU6}}$

$\Delta \Delta$ Ct Sample $=\Delta \mathrm{Ct}$ Sample $-\Delta \mathrm{Ct}$ Control Mean

Relative quantitation (Fold Change) of sample $=$ $2-\Delta \Delta C t$

\section{Statistical analysis}

Statistical analysis were performed using SPSS version 21.0(IBM-SPSS, Chicago, IL, USA). Data were presented as mean $\pm S D$, median and percentages. Pearson's $\chi 2$ test was employed to analyze categorical data as appropriate. For data that did not follow normal distribution Kruskal Wallis test was performed. Receiving operation characteristic (ROC) curve (analyzed as area under the curve (AUC), standard error (SE) and 95\% confidence interval (CI)) was constructed to detect the validity and cut off value of tumor biomarkers (CEA, CA 15-3 and microRNA-27a) for discrimination between malignant cases and nonmalignant cases. Validity statistics (sensitivity, specificity, accuracy, positive and negative predictive value PPV \& NPV) were calculated. $P$ value $\leq 0.05$ was considered significant.

\section{Results}

In the current study, the expression level of miR-27a and tumor markers (CEA and CA 15-3) were detected among the five investigated groups. The demographic and clinical data are reported in table 1.

Table 1. Demographic and clinical data in the studied groups

\begin{tabular}{lccccc} 
Parameter & $\begin{array}{c}\text { Control } \\
(n=15)\end{array}$ & $\begin{array}{c}\text { High Risk } \\
(n=20)\end{array}$ & $\begin{array}{c}\text { Benign } \\
(n=20)\end{array}$ & $\begin{array}{c}\text { Stage I \& II } \\
(n=20)\end{array}$ & $\begin{array}{c}\text { Stage III \& IV } \\
(n=20)\end{array}$ \\
\hline Age & $42.33 \pm 11.6$ & $35.70 \pm 6.2$ & $39.80 \pm 9.6$ & $48.10 \pm 9.1$ & $57.55 \pm 9.6$ \\
\hline $\begin{array}{l}\text { Marital Status } \\
\text { Married }\end{array}$ & $1(6.7 \%)$ & $3(15 \%)$ & $5(25 \%)$ & $5(25 \%)$ & $4(20 \%)$ \\
$\quad \begin{array}{l}\text { Unmarried } \\
\text { Breast feeding }\end{array}$ & $14(93.3 \%)$ & $17(85 \%)$ & $15(75 \%)$ & $15(75 \%)$ & $16(80 \%)$ \\
$\quad$ No & $3(20 \%)$ & $4(20 \%)$ & $4(20 \%)$ & $4(20 \%)$ & $2(10 \%)$ \\
$\quad$ Yes & $12(80 \%)$ & $16(80 \%)$ & $16(80 \%)$ & $16(80 \%)$ & $18(90 \%)$ \\
\hline $\begin{array}{l}\text { Menopausal status } \\
\text { Pre-menopausal }\end{array}$ & $10(66.7 \%)$ & $20(100 \%)$ & $16(80 \%)$ & $13(65 \%)$ & $6(30 \%)$ \\
post-menopausal & $5(33.3 \%)$ & - & $4(20 \%)$ & $7(35 \%)$ & $14(70 \%)$
\end{tabular}

Mean rank levels of CEA, CA 15-3and miR-27a are reported in table 2.There was statistically significant elevation of serum CEA and CA 15-3 levels in patients with benign breast lesions compared to control group. Also, there was statistically significant elevation of serum CEA and CA 15-3 levels in BC patients compared to control group, high risk group and patients with 
benign breast lesions. Serum CEA and CA 15-3 levels were significantly higher in late BC (TNM stages III \& IV) compared to early BC (TNM stages I \& II).

There was no statistically significant difference of serum miR-27a levels in high risk group compared to control group $(P=0.714)$. Also, there was no statistically significant difference of serum miR-27a levels in benign group compared to both control group $(P=$ $0.454)$ and high-risk group $(p=0.679)$. There was statistically significant elevation of serum miR27a levels in BC patients compared to control group, high risk group and patients with benign breast lesions with $P<0.001$ for each.

In the present study plasma miR-27a levels showed a positive association with the TNM stages of BC. Plasma miR-27a was significantly higher in late BC (TNM stages III \& IV) compared to early BC (TNM stages I \& II) with ( $P$-value < 0.001).

Table 2.Comparison of tumor biomarkers expression level between Groups

\begin{tabular}{|c|c|c|c|c|c|c|}
\hline Parameter & $\begin{array}{l}\text { Control } \\
(n=15)\end{array}$ & $\begin{array}{l}\text { High risk } \\
(n=20)\end{array}$ & $\begin{array}{l}\text { Benign } \\
(n=20)\end{array}$ & $\begin{array}{c}\text { Early BC } \\
\text { (Stage I\&II) } \\
(\mathrm{N}=20)\end{array}$ & $\begin{array}{c}\text { Late BC } \\
\text { (Stage III\&IV) } \\
(\mathrm{N}=20)\end{array}$ & $P$-value \\
\hline \multicolumn{7}{|l|}{ CEA } \\
\hline Mean \pm SD & $1.33 \pm 1.1$ & $1.94 \pm 1.4$ & $3.42 \pm 2.5$ & $6.9 \pm 3.1$ & $11.81 \pm 3.7$ & \multirow{4}{*}{$<0.001^{*}$} \\
\hline Median & 1.0 & 1.7 & 2.9 & 7.5 & 11.4 & \\
\hline \multirow[t]{2}{*}{$P$-value** } & $1 \mathrm{vs} .2=\mathrm{NS}$ & $2 \mathrm{vs} .3=\mathrm{NS}$ & $3 v s .4<0.001$ & 1 vs. $4<0.001$ & 1 vs. $5<0.001$ & \\
\hline & 1 vs. $3=0.021$ & $2 v s .4<0.001$ & $3 v s .5<0.001$ & 4 vs. $5<0.001$ & 2 vs. $5<0.001$ & \\
\hline
\end{tabular}

CA 15-3

$\begin{array}{lcccccc}\text { Mean } \pm \text { SD } & 10.71 \pm 6.2 & 15.23 \pm 8.2 & 42.57 \pm 20.4 & 68.51 \pm 11.7 & 101.57 \pm 41.9 & \\ \text { Median } & 10 & 13 & 44 & 68 & 98 & <0.001^{*} \\ P \text { - value** } & 1 \text { vs. } 2=\text { NS } & 2 \text { vs. } 3<0.001 & 3 \text { vs. } 4<0.001 & 1 \text { vs. } 4<0.001 & 1 \text { vs. } 5<0.001 & \\ & 1 \text { vs. } 3<0.001 & 2 \text { vs. } 4<0.001 & 3 \text { vs. } 5<0.001 & 4 \text { vs. } 5<0.001 & 2 \text { vs. } 5<0.001 & \end{array}$

MiR-27a

$\begin{array}{lcccccc}\text { Mean } \pm \text { SD } & 1.00 \pm 0.5 & 1.53 \pm 1.4 & 2.08 \pm 1.9 & 8.20 \pm 3.9 & 20.78 \pm 7.9 & \\ \text { Median } & 1.0 & 1.2 & 1.8 & 8.3 & 19.0 & <0.001^{*} \\ P \text { - value** } & 1 \text { vs. } 2=\text { NS } & 2 \text { vs. } 3=\text { NS } & 3 \text { vs. } 4<0.001 & 1 \text { vs. } 4<0.001 & 1 \text { vs. } 5<0.001 & \\ & 1 \text { vs. } 3=\text { NS } & 2 \text { vs. } 4<0.001 & 3 \text { vs. } 5<0.001 & 4 \text { vs. } 5<0.001 & 2 \text { vs. } 5<0.001 & \end{array}$

*Kruskal Wallis test was used to compare the median difference between groups.

** Post-hoc test with Bonferroni corrections. $\mathrm{P}>0.05$ is not significant (NS).

Diagnostic performance of $B C$ biomarkers for malignant cases (Control, High risk group, Benign cases VS malignant Cases) (table 3, figure 1)

In this study, we constructed ROC curve to assess the performance of BC biomarkers in discriminating malignant form nonmalignant individuals included in our study (healthy controls, high risk group and benign cases) they were added together as one group. By plotting the ROC, the diagnostic efficacy was determined using the calculated cutoff point as $5 \mathrm{ng} / \mathrm{ml}, 58$ $\mathrm{U} / \mathrm{ml}$ and 2.5-fold change for CEA, CA15-3and miR-27a. AUC was 0.943, 0.955 and 0.983, respectively. Considering these cutoff points; CEA was $87.5 \%$ accurate, $87.5 \%$ sensitive and $87 \%$ specific with positive predictive value $87 \%$ 
and negative predictive value $87.5 \%$. CA $15-3$ was $87 \%$ accurate, $85 \%$ sensitive and $89 \%$ specific with positive predictive value $88.5 \%$ and negative predictive value $85.5 \%$. miR-27a was 94\% accurate, $97.5 \%$ sensitive and $91 \%$ specific with positive predictive value $91.5 \%$ and negative predictive value $97.3 \%$.

Table 3. Performance of the tumor biomarkers in discrimination between $\mathrm{BC}$ cases and nonmalignant cases.

\begin{tabular}{lccc} 
& \multicolumn{3}{c}{ Diagnostic criteria } \\
& CEA & CA 15-3 & MiR-27a \\
\hline AUC & 0.943 & 0.955 & 0.983 \\
Cut off & $5 \mathrm{ng} / \mathrm{ml}$ & $58 \mathrm{U} / \mathrm{ml}$ & $2.5 \mathrm{FC}$ \\
Accuracy & $87.5 \%$ & $87 \%$ & $94 \%$ \\
Sensitivity \% & $87.5 \%$ & $85 \%$ & $97.5 \%$ \\
Specificity \% & $87 \%$ & $89 \%$ & $91 \%$ \\
PPV \% & $87 \%$ & $88.5 \%$ & $91.5 \%$ \\
NPV \% & $87.5 \%$ & $85.5 \%$ & $97.3 \%$ \\
\hline
\end{tabular}

The cutoff values were $5 \mathrm{ng} / \mathrm{ml}, 58 \mathrm{U} / \mathrm{ml}$ and $2.5 \mathrm{FC}$ for CEA, CA 15-3 and miR-27a respectively.

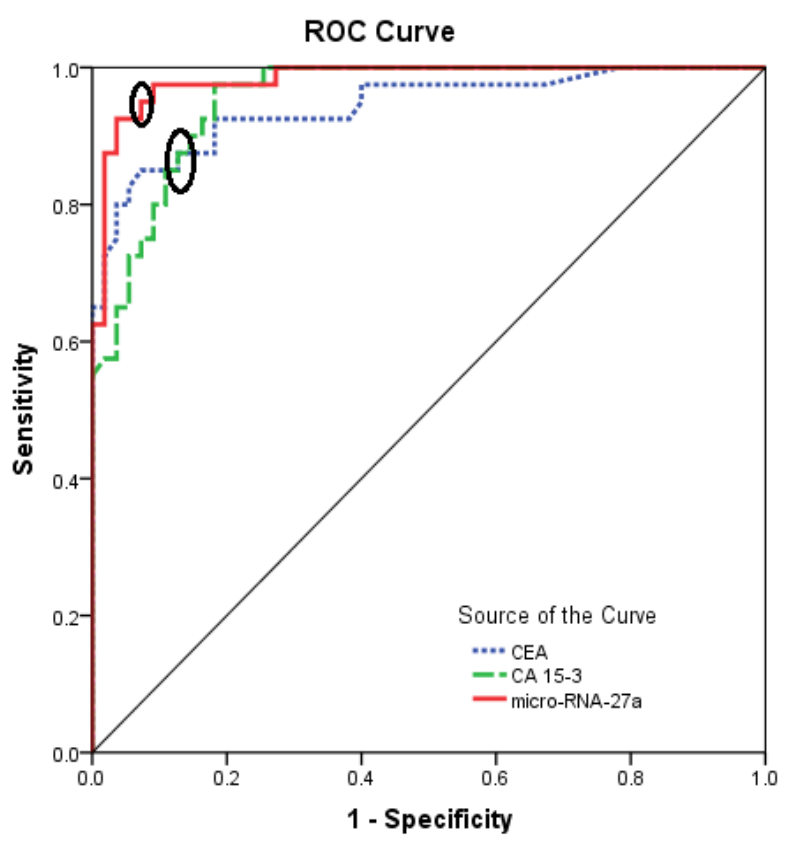

Figure 1. ROC curve for tumor biomarkers in discrimination between $B C$ cases and nonmalignant cases. The AUC was 0.943, 0.955 and 0.983 for CEA, CA15-3 and miR-27a, respectively. Cutoff values were $5 \mathrm{ng} / \mathrm{ml}, 58 \mathrm{U} / \mathrm{ml}$ and $2.5 \mathrm{FC}$ respectively. Open circles donate best cutoff points.

\section{Discussion}

Early detection of $B C$ requires a non-invasive and sensitive marker to detect molecular differences associated with carcinogenesis and hence properly directing patients at high risk for optimal clinical management. Routine tumor markers CEA and CA15.3 which are common for $B C$ diagnosis and prognosis, were reported to elevate in patients with benign breast lesions although could not detect those at high risk. ${ }^{28}$

The mature miR-27a forms one strand of the RNA duplex. It was involved in BC, in which elevated miR-27a expression increased the percentage of cells in G2/M stage, resulting in an oncogenic function. ${ }^{29}$ MiR-27a has shown good potential for therapeutic applications. ${ }^{30}$ Its role has been well studied in $\mathrm{BC}$ as a biomarker for diagnosis and prognosis. ${ }^{31}$

Our study revealed that serum CA 15-3 and CEA were significantly higher in late $B C$ patients compared to early cancer patients, which in turn were significantly higher than patients with benign breast lesions, high risk group of patients and healthy controls. Also they were significantly higher in patients with benign breast lesions compared to healthy controls. CA 15-3 was significantly higher in patients with benign breast lesions compared to the high risk group. There was no statistical significant difference in CEA level between patients with benign breast lesions and the high risk group. There was no statistical significant difference in levels of both CA 15-3 and CEA between both the control group and the high risk group.

Our results were consistent with the results of Magid et al ${ }^{32}$ who reported that the mean serum CA 15-3 and CEA were significantly higher in patients with $B C$ as compared to control groups with $\mathrm{P}$ value $<0.0001$. Also our results are parallel to the study of Zaleski et $\mathrm{al}^{33}$ who observed that the tumor markers CEA and CA 15-3 were significantly higher in serum of $B C$ patients as compared with healthy women.

Our results were also consistent with that of Fang et al ${ }^{34}$ who observed that late-stage cancer patients revealed significantly higher levels of CEA and CA15-3 compared with earlystage ones, suggesting that the serum levels of these tumor markers might be more efficient 
for monitoring advanced tumors than early diagnosis.

Lian et al, 2019 reported that serum CEA and CA15-3 of patients with BC were higher than those of healthy volunteer group and patients with benign breast diseases $(P<0.05$ for each). ${ }^{35}$ This is parallel to our study which we found that serum CA 15-3 and CEA levels were significantly higher in patients with $B C$ than those with benign breast lesions and healthy control group. Similar to these results is the study of Dolscheid-Pommerich et al, who reported that both CA 15-3 and CEA were significantly higher in patients with $B C$ compared to patients with benign diseases with $P$ value 0.022 and 0.019 respectively. ${ }^{36}$

In this study the serum levels of CA 15-3 and CEA were significantly higher in patients with benign breast lesions compared to healthy controls. This is consistent with that of Youssef et al who found that serum CEA and CA 15-3 levels were statistically significantly higher in both benign and BC subjects compared to control group. ${ }^{37}$

This present study exhibited significantly higher level of plasma miR-27a in BC patients compared to both healthy control group and the high-risk group. It was also significantly higher in BC patients compared to patients with benign breast lesions. Our results were consistent with the results of a study done by Swellam et $\mathrm{al}^{26}$ in which miR-27a expression level was determined among three groups: BC patients, patients with benign $\mathrm{BC}$ patients and healthy controls, and its expression level was significantly higher in primary BC patients followed by benign breast patients while its level among the healthy individuals was the lowest.

A study done by Li et $\mathrm{al}^{38}$ found that miR-27a is significantly upregulated in BC tissues compared to tissues from normal breast and it was correlated with poor survival outcome and tumor progression, this was in agreement with our study. Tang et al reported thatmiR-27a was markedly up-regulated in invasive breast cancers. They found that miR-27a was higher expressed in breast invasive cancers with distant metastasis, compared with nonmetastatic cancers. ${ }^{39}$ Raeisi et al revealed the prognostic significance of miR-27a overexpression in various carcinomas, such as gastric cancer, acute lymphoblastic leukemia and osteosarcoma. This indicated that the breast-invasive cancers with higher miR-27a expression tended to have distant metastasis. ${ }^{23}$

We have assessed the miR-27a level in different stages of BC. Our results revealed that miR-27a significantly higher in late BC compared to early cancer. This was consistent with the study done by Swellam et al which reported significant link between expression of miR-27a and the clinical stages of $\mathrm{BC} .^{26}$

A study done by Tang et al ${ }^{39}$ reported that the expression of miR-27a is strongly correlated with the clinical stages and overall survival times of patients with BC. Raeisi et al also reported that overexpression of miR-27a was associated with shorter disease-free survival and the overall survival of BC patients. His study suggested that miR-27a expression was an independent prognostic factor for BC progression. ${ }^{23}$

In our present study there was no significant difference between the level of miR-27a between patients with benign breast lesions and both high risk group and the control group. Also, there was no significant difference between the level of miR-27a between high risk group and the control group. This suggests that mir-27a serves as an oncogenic microRNA which is cannot be detected in benign conditions or healthy individuals at high risk of developing BC.

In the present study, we evaluated the diagnostic performance of plasma miR-27a and common routine markers for discriminating $B C$ cases from healthy controls, high risk group and patients with benign breast lesions. We plotted the ROC curve and miR- 27a was superior to both routine markers CEA and CA 15-3 for diagnosis for BC. Our results showed plasma miR-27a was more sensitive (97.5\%) with AUC of 0.983 , than serum CEA which was $87.5 \%$ sensitive with AUC of 0.943 and serum CA15-3 which was $87 \%$ sensitive with AUC of 0.955 . Our results were in agreement with Swellam et al. who reported that serum miR-27a had sensitivity $92 \%$, specificity $92 \%$ and AUC of 0.955 . $^{26}$ 
It is concluded that measuring miR-27a in peripheral blood may be a promising tool for detection of early stages of BC. Plasma miR-27a level may have a potential value for discrimination of malignant cases from nonmalignant cases with better diagnostic performance than serum CEA and CA 15-3. Further studies with larger number of patients will offer more detailed knowledge about the diagnostic role of plasma miR-27a in BC diagnosis and its role in diagnosis of benign breast lesions and prediction of $B C$ in females with risk factors for getting $B C$.

\section{Author Contributions}

MIS, methodology, analysis and interpretation of data and writing, review and editing. OO, project administration, conception and design of the study. and supervision. DAN, methodology, Analysis and interpretation of data. MAJ, review and editing, clinical evaluation of patients and resources. EMA, writing the original draft, review and editing and practical work.

\section{Declaration of Conflicting Interests}

The author(s) declared no potential conflicts of interest with respect to the research, authorship, and/or publication of this article.

\section{Funding}

This research did not receive any specific grant from funding agencies in the public, commercial, or notfor-profit sectors

\section{Ethical approval}

The study protocol was reviewed and approved by the Committee of Medical Ethics, Faculty of Medicine, Assiut University.

\section{Informed consent}

A signed consent form was obtained from each study participant.

\section{References}

1. Dolatkhah, R., M.H. Somi, M.A. Jafarabadi, et al., (2020) Breast Cancer Survival and Incidence: 10 Years Cancer Registry Data in the Northwest, Iran. International Journal of Breast Cancer, 2020: p. 1963814.
2. Fitzmaurice, C., T.F. Akinyemiju, F.H. Al Lami, et al., (2018) Global, regional, and national cancer incidence, mortality, years of life lost, years lived with disability, and disability-adjusted life-years for 29 cancer groups, 1990 to 2016: a systematic analysis for the global burden of disease study. JAMA oncology, 4(11): p. 1553-1568.

3. Gewaifel, G.I., M.M. Bahnasy, I. Kharboush, et al., (2019) Geospatial Analysis of Breast Cancer in Alexandria: Application of a Novel Public Health Tool. Egyptian Journal of Community Medicine, 37(2).

4. El-Nasr, E.M.S., (2017) Breast Cancer risk factors and screening practices Among Women Attending Family Health Centers in Cairo Governorate. Breast, 20: p. 8.

5. Migowski, A., (2015) [Early detection of breast cancer and the interpretation of results of survival studies]. Cien Saude Colet, 20(4): p. 1309.

6. Onega, T., L.E. Goldman, R.L. Walker, et al., (2016) Facility Mammography Volume in Relation to Breast Cancer Screening Outcomes. J Med Screen, 23(1): p. 31-7.

7. Ozmen, N., R. Dapp, M. Zapf, et al., (2015) Comparing different ultrasound imaging methods for breast cancer detection. IEEE Trans Ultrason Ferroelectr Freq Control, . 62(4): p. 637-46.

8. Roganovic, D., D. Djilas, S. Vujnovic, et al., (2015) Breast MRI, digital mammography and breast tomosynthesis: comparison of three methods for early detection of breast cancer. Bosn J Basic Med Sci, 15(4): p. 64-8.

9.Yen, T.W., J. Li, R.A. Sparapani, et al., (2016) The interplay between hospital and surgeon factors and the use of sentinel lymph node biopsy for breast cancer. Medicine, 95(31).

10. Cheng, B., (2016) of a chemiluminescent immunoassay for cancer antigen 15-3. Labeled Immunoass. Clin. Med, 23: p. 1348-1351.

11. Mirabelli, P. and M. (2013) Incoronato, Usefulness of traditional serum biomarkers for management of breast cancer patients. Biomed Res Int, 2013: p. 685641.

12. Tahiri, A., M.R. Aure, and V.N. Kristensen, (2018) MicroRNA networks in breast cancer cells, in Cancer Systems Biology. Springer. p. 55-81.

13. Ortiz-Quintero, B., (2016) Cell-free microRNAs in blood and other body fluids, as cancer biomarkers. Cell Proliferation, 49(3): p. 281-303.

14. Condrat, C.E., D.C. Thompson, M.G. Barbu, et al., (2020) miRNAs as Biomarkers in Disease: Latest Findings Regarding Their Role in Diagnosis and Prognosis. Cells, 9(2). 
15. Teoh, S.L. and S. Das, (2017) The Role of MicroRNAs in Diagnosis, Prognosis, Metastasis and Resistant Cases in Breast Cancer. Curr Pharm Des, 23(12): p. 1845-1859.

16. Zhang, J., Z. Cao, G. Yang, et al., (2019) MicroRNA-27a (miR-27a) in Solid Tumors: A Review Based on Mechanisms and Clinical Observations. Front Oncol, 9: p. 893.

17. Ding, L., S. Zhang, M. Xu, et al., (2017) MicroRNA27a contributes to the malignant behavior of gastric cancer cells by directly targeting $\mathrm{PH}$ domain and leucine-rich repeat protein phosphatase 2. Journal of Experimental \& Clinical Cancer Research, 36(1): p. 45.

18. Zheng, X., F. Zhang, Y. Zhao, et al., (2018) Selfassembled dual fluorescence nanoparticles for CD44targeted delivery of anti-miR-27a in liver cancer theranostics. Theranostics, 8(14): p. 3808.

19. Shishodia, G., G. Verma, B.C. Das, et al., (2018) miRNA as viral transcription tuners in HPV-mediated cervical carcinogenesis. Front Biosci (Schol Ed), 10: p. 21-47.

20.Xiao, F., Y. Li, Y. Wan, et al., (2018) MircroRNA139 sensitizes ovarian cancer cell to cisplatin-based chemotherapy through regulation of ATP7A/B. Cancer chemotherapy and pharmacology, 81(5): p. 935-947.

21. Wan, X., W. Huang, S. Yang, et al., (2016) Androgen-induced miR-27A acted as a tumor suppressor by targeting MAP2K4 and mediated prostate cancer progression. The international journal of biochemistry \& cell biology, 79: p. 249-260.

22. Abbasi, B., J. Iqbal, T. Mahmood, et al., (2018) Role of dietary phytochemicals in modulation of miRNA expression: Natural swords combating breast cancer. Asian Pacific Journal of Tropical Medicine, 11(9): p. 501-509.

23. Raeisi, F., E. Mahmoudi, M. Dehghani-Samani, et al., (2020) Differential Expression Profile of miR-27b, miR-29a, and miR-155 in Chronic Lymphocytic Leukemia and Breast Cancer Patients. Molecular Therapy-Oncolytics, 16: p. 230-237.

24. Edge, S.B., D.R. Byrd, M.A. Carducci, et al., (2010) AJCC cancer staging manual. Vol. 649. Springer New York.

25. Edge SB, B.D.a.C.C., (2010) American Joint Committee on cancer (AJCC) Breast. In AJCC Cancer Staging Manual, 7th ed. New York, NY: Springer, p. pp 347-76.

26. Swellam, M., R.F. Zahran, S.A. Ghonem, et al., (2019) Serum MiRNA-27a as potential diagnostic nucleic marker for breast cancer. Archives of Physiology and Biochemistry, p. 1-7.
27. Livak, K.J. and T.D. (2001) Schmittgen, Analysis of relative gene expression data using real-time quantitative PCR and the 2- $\triangle \triangle C T$ method. methods, 25(4): p. 402-408.

28. Cardoso, F., M. Saghatchian, and A. Thompson, (2008) Inconsistent criteria used in American Society of Clinical Oncology 2007 update of recommendations for the use of tumor markers in breast cancer. Journal of clinical oncology, 26(12): p. 2058-2059.

29. Mertens-Talcott, S.U., S. Chintharlapalli, X. Li, et al., (2007) The oncogenic microRNA-27a targets genes that regulate specificity protein transcription factors and the G2-M checkpoint in MDA-MB-231 breast cancer cells. Cancer research, 67(22): p. 11001-11011.

30. Fletcher, C.E., D.A. Dart, A. Sita-Lumsden, et al., (2012) Androgen-regulated processing of the oncomir miR-27a, which targets Prohibitin in prostate cancer. Human molecular genetics, 21(14): p. 3112-3127.

31. Lü, L., X. Mao, P. Shi, et al., (2017) MicroRNAs in the prognosis of triple-negative breast cancer: A systematic review and meta-analysis. Medicine, 96(22)

32. Magid, R., A.R.H. Al-Salih, and H. Al-Hilaly, (2017) Immunological study of CA-15.3 and CEA tumor markers among post-operative breast cancer patients. Al-Qadisiyah Medical Journal,. 13(24): p. 94-102.

33. Zaleski, M., M. Kobilay, L. Schroeder, et al., (2018) Improved sensitivity for detection of breast cancer by combination of miR-34a and tumor markers CA 15-3 or CEA. Oncotarget,. 9(32): p. 22523.

34. Fang, C., Y. Cao, X. Liu, et al., (2017) Serum CA125 is a predictive marker for breast cancer outcomes and correlates with molecular subtypes. Oncotarget, 8(38): p. 63963-63970.

35. Lian, M., C. Zhang, D. Zhang, et al., (2019) The association of five preoperative serum tumor markers and pathological features in patients with breast cancer. J Clin Lab Anal, 33(5): p. e22875.

36. Dolscheid-Pommerich, R.C., M. Keyver-Paik, T. Hecking, et al., (2017) Clinical performance of $\mathrm{LOCl}^{\mathrm{TM}}$ based tumor marker assays for tumor markers CA 15-3, CA 125, CEA, CA 19-9 and AFP in gynecological cancers. Tumour Biol, 39(10): p. 1010428317730246.

37. Youssef, S.S., M.M. Mohammad, and L.R. Ezz-ElArab, (2015) Clinical Significance of Serum IL-12 Level in Patients with Early Breast Carcinoma and Its Correlation with Other Tumor Markers. Open Access Maced J Med Sci, 3(4): p. 640-4. 
38. Li, W., Z. Yu, and B. Ma, (2018) The increase of miR-27a affects the role of cisplatin on proliferation and migration capacities of liver cancer cells. European review for medical and pharmacological sciences, 22(17): p. 5490-5498.
39. Tang, W., J. Zhu, S. Su, et al., (2012) MiR-27 as a prognostic marker for breast cancer progression and patient survival. PLoS One, 7(12): p. e51702. 